\title{
ARTICLE
}

Received 19 Aug 2016 | Accepted 5 Apr 2017 | Published 24 May 2017

DOl: $10.1038 /$ ncomms15548

OPEN

\section{The interfacial structure of water droplets in a hydrophobic liquid}

Nikolay Smolentsev ${ }^{1}$, Wilbert J. Smit ${ }^{2}$, Huib J. Bakker ${ }^{2} \&$ Sylvie Roke ${ }^{1}$

Nanoscopic and microscopic water droplets and ice crystals embedded in liquid hydrophobic surroundings are key components of aerosols, rocks, oil fields and the human body. The chemical properties of such droplets critically depend on the interfacial structure of the water droplet. Here we report the surface structure of $200 \mathrm{~nm}$-sized water droplets in mixtures of hydrophobic oils and surfactants as obtained from vibrational sum frequency scattering measurements. The interface of a water droplet shows significantly stronger hydrogen bonds than the air/water or hexane/water interface and previously reported planar liquid hydrophobic/water interfaces at room temperature. The observed spectral difference is similar to that of a planar air/water surface at a temperature that is $\sim 50 \mathrm{~K}$ lower. Supercooling the droplets to $263 \mathrm{~K}$ does not change the surface structure. Below the homogeneous ice nucleation temperature, a single vibrational mode is present with a similar mean hydrogen-bond strength as for a planar ice/air interface.

\footnotetext{
${ }^{1}$ Laboratory for Fundamental BioPhotonics (LBP), Institute of Bioengineering (IBI), and Institute of Materials Science (IMX), School of Engineering (STI), and Lausanne Centre for Ultrafast Science (LACUS), École Polytechnique Fédérale de Lausanne (EPFL), CH-1015 Lausanne, Switzerland. ${ }^{2}$ AMOLF, Science Park 104, 1098 XG Amsterdam, The Netherlands. Correspondence and requests for materials should be addressed to S.R. (email: sylvie.roke@epfl.ch).
} 
A queous hydrophobic interfaces play a defining role on nano- and microscopic length scales. Pockets and droplets of water in a hydrophobic environment are omnipresent in the atmosphere (as ice particles and cloud droplets ${ }^{1}$ ), the earth ${ }^{2,3}$ (in oil fields and inside internal pores of many geological materials), and in chemical ${ }^{4,5}$ and biological processes $^{6-8}$ (as vehicles for medicine delivery ${ }^{9}$ ). For all of these processes, the nanoscopic water droplet/hydrophobic interface plays a critical role in determining the fate of the system. The reactivity of confined water is crucial for atmospheric science $^{1}$, earth science ${ }^{3}$ and biology ${ }^{10}$. The molecular surface properties of water droplets are commonly inferred from data obtained from the macroscopic air/water interface ${ }^{11-16}$ or from aqueous solutions of solvated hydrophobes ${ }^{17}$, systems that are very different in both their size, chemical composition and temperature dependence from actual droplet surfaces. As such, the surface structure and reactivity of water droplets and confined water remains elusive, despite its relevance.

Here we present temperature-controlled sum frequency scattering (SFS) experiments on nanoscopic water droplets in a liquid hydrophobic environment. We find that compared to planar water/air or water/hexane interfaces the spectrum reveals a greater order corresponding to that of a planar water surface at a $\sim 50$ or $40 \mathrm{~K}$ lower temperature. This increase in order is explained by the formation of an extended hydrophobic network, which does not exist on planar interfaces and in solution. In addition, on supercooling the droplets, the surface spectrum does not change shape. Cooling the water droplets below the homogeneous ice nucleation temperature results in a spectrum with a single symmetric peak that is similar to the one found for the basal ice/air interface.

\section{Results}

Room temperature droplet experiments. Nanoscopic (100 nm radius) water droplets are prepared via sonication in $\mathrm{d}_{34}$-hexadecane (Fig. 1) or in a 1:1 mixture of decane and cyclohexane (Fig. 2), both with $5 \mathrm{mM}$ of a hydrophobic surfactant molecule that partially covers the interface (Span80, Fig. 1a). The water-hexadecane partitioning coefficient for these hydrophobic liquids is $5.5 \times 10^{-4}$ (Span80 (ref. 18)), $>1.6 \times 10^{-6}$ (decane) and $1.6 \times 10^{-4}$ (cyclohexane) ${ }^{19}$, providing a liquid hydrophobic/water interface in all systems. The interfacial structure of the water droplets and ice nanocrystals is measured with vibrational $\mathrm{SFS}^{20,21}$, a method that combines interfacespecific sum frequency generation (SFG) $11,12,14,15,22,23$ with light scattering $^{24}$ (illustrated in Fig. 1b). The scattered vibrational spectra report on the average orientational order and $\mathrm{H}$-bond network structure of water molecules in the first few monolayers of water at the interfacial region ${ }^{21}$.

Figure $1 \mathrm{c}$ shows the scattered SF spectrum of $\mathrm{D}_{2} \mathrm{O}$ nanodroplets in oil $(296 \mathrm{~K})$ and reflection SFG spectra of a planar liquid $\mathrm{D}_{2} \mathrm{O}$ /air and a planar $\mathrm{D}_{2} \mathrm{O} /$ hexane interface. The spectra were normalized taking into account linear absorption effects as well as discontinuities in the normal component of the electromagnetic fields as they cross the droplet interface (see Methods section). The SFG spectra of the two planar interfaces of Fig. 1c have three main features: A peak at $2,370 / 2,400 \mathrm{~cm}^{-1}$, a peak at $2,500 \mathrm{~cm}^{-1}$ and a peak at $2,745 / 2,725 \mathrm{~cm}^{-1}$. The frequency of the OD stretching vibrations is correlated to the strength of the interfacial hydrogen $(\mathrm{H})$-bond interaction: the OD frequency decreases with increasing $\mathrm{H}$-bond strength. Hence, the feature at $2,500 \mathrm{~cm}^{-1}$ reports on water molecules that are more weakly $\mathrm{H}$-bonded than the ones that attribute to the feature at $2,370 / 2,400 \mathrm{~cm}^{-1}$. The peak at $2,745 / 2,725 \mathrm{~cm}^{-1}$ originates from interfacial OD bonds that are not H-bonded ${ }^{25}$. The $296 \mathrm{~K}$ droplet/hydrophobic interface spectrum contains the same 2,370 and $2,500 \mathrm{~cm}^{-1}$ features (indicated by the dotted lines), but the higher frequency components are significantly less intense than they are in the planar interface spectra. This indicates that there are relatively more strongly $\mathrm{H}$-bonded water molecules at the water droplet interface than at the air/water and hexane/water interface recorded at the same temperature. The SFG spectrum of the Span80/water interface is similar to that of $\mathrm{D}_{2} \mathrm{O} /$ hexane and $\mathrm{D}_{2} \mathrm{O} /$ air and shown in the Supplementary Fig. 1.

The peak ratio of the 2,370 and $2,500 \mathrm{~cm}^{-1}$ modes is temperature dependent. This temperature-dependent ratio is plotted in Fig. 1d for the air/water and hexane/water interfaces. Lowering the temperature increases ${ }^{26}$ the ratio, indicating that the population of stronger $\mathrm{H}$-bonds increases over that of weaker $\mathrm{H}$-bonds. The peak ratio obtained for the water droplets is also indicated (black marker). Extrapolating the ratios found for the air/water and hexane/water spectra (blue and red lines) the same ratio as for the water droplets would be found at a hypothetical planar water/air or water/hexane interface of $\sim 245 / 260 \mathrm{~K}$ (that is, $\sim 50 / 40 \mathrm{~K}$ colder). Thus, the water molecules of the water droplet/hydrophobic interface appear to be much more structured than the water molecules at a planar water/air or water/hexane interface. Note that this conclusion was arrived at by comparing amplitudes at particular frequencies. The spectrum of water is known to be highly complex, involving strong inhomogeneous broadening 27,28 , excitonic coupling effects $^{29}$ and a Fermi resonance with the overtone of the bending vibration ${ }^{30}$. As such, aside from the feature that high-frequency modes generally refer to more weakly $\mathrm{H}$-bonded water molecules than low-frequency modes, there are many ways to interpret the spectral information ${ }^{15,16,25,26,28,31-35}$. Our analysis addresses the temperature dependence of the ratio of weakly and strongly $\mathrm{H}$-bonded water molecules, and is as such quite model independent.

Very similar SFS spectra are obtained for other combinations of hydrophobic oils (mixtures of decane and cyclohexane, fluorinated oil) and surfactants (Tween80, tri-block PEG 900, Supplementary Fig. 2), which indicates that the observed red-shift of the spectrum is a common phenomenon for water droplets in various hydrophobic liquids. The absence of unbonded OD groups in the droplet spectrum can be partially explained by the presence of $\mathrm{OH}$ groups on the Span 80 molecule that can form $\mathrm{H}$-bonds with interfacial water molecules that would otherwise be unbonded (see Supplementary Note 1, Supplementary Fig. 3 and Supplementary Table 1). As a result, the peak at $2,745 \mathrm{~cm}^{-1}$, corresponding to OD groups that are not $\mathrm{H}$-bonded, vanishes. From the detection limit of the SFS system ${ }^{36}$, it follows that the imbalance between OD groups that point in or out of the water droplet surface is less than one free OD group per $27 \mathrm{~nm}^{2}$. The difference with the droplet spectrum probably arises from a difference in structure, which we will discuss in more detail further on. Note that impurities are not expected to be a problem here $^{36}$ as the sample volume is small $(\sim 50 \mu \mathrm{l})$, and the surface to volume ratio is large $\left(\sim 10^{5} \mathrm{~cm}^{-1}\right.$; which is at least three orders of magnitude larger than in standard planar reflection SFG measurements).

Supercooling the droplets. Next, we repeat the experiment at reduced temperatures, supercooling the droplets (Fig. 2a) and ultimately freezing them by cooling below the homogeneous nucleation point of $\mathrm{D}_{2} \mathrm{O}(237 \mathrm{~K}$ (ref. 37$)$, Fig. $\left.2 \mathrm{~b}\right)$. Figure $2 \mathrm{a}$ shows SFS spectra recorded at different temperatures $(296 \mathrm{~K}$, red, $273 \mathrm{~K}$, green and $263 \mathrm{~K}$, blue) using a mixture of decane and cyclohexane as the main phase. The droplet spectrum from Fig. $1 \mathrm{c}$ is also shown (black line). It can be seen that despite the different 


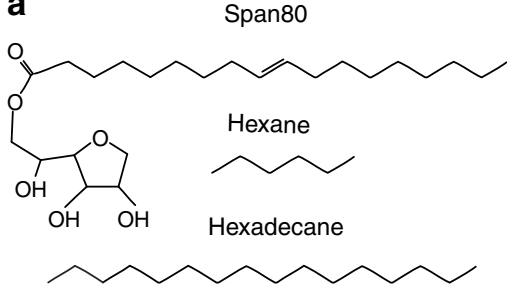

b
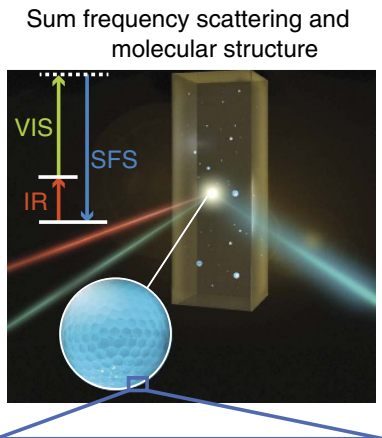

C IR frequency for $\mathrm{H}_{2} \mathrm{O}\left(\mathrm{cm}^{-1}\right)$

$\begin{array}{lllllll}3,100 & 3,200 & 3,300 & 3,400 & 3,500 & 3,600 & 3,700\end{array}$
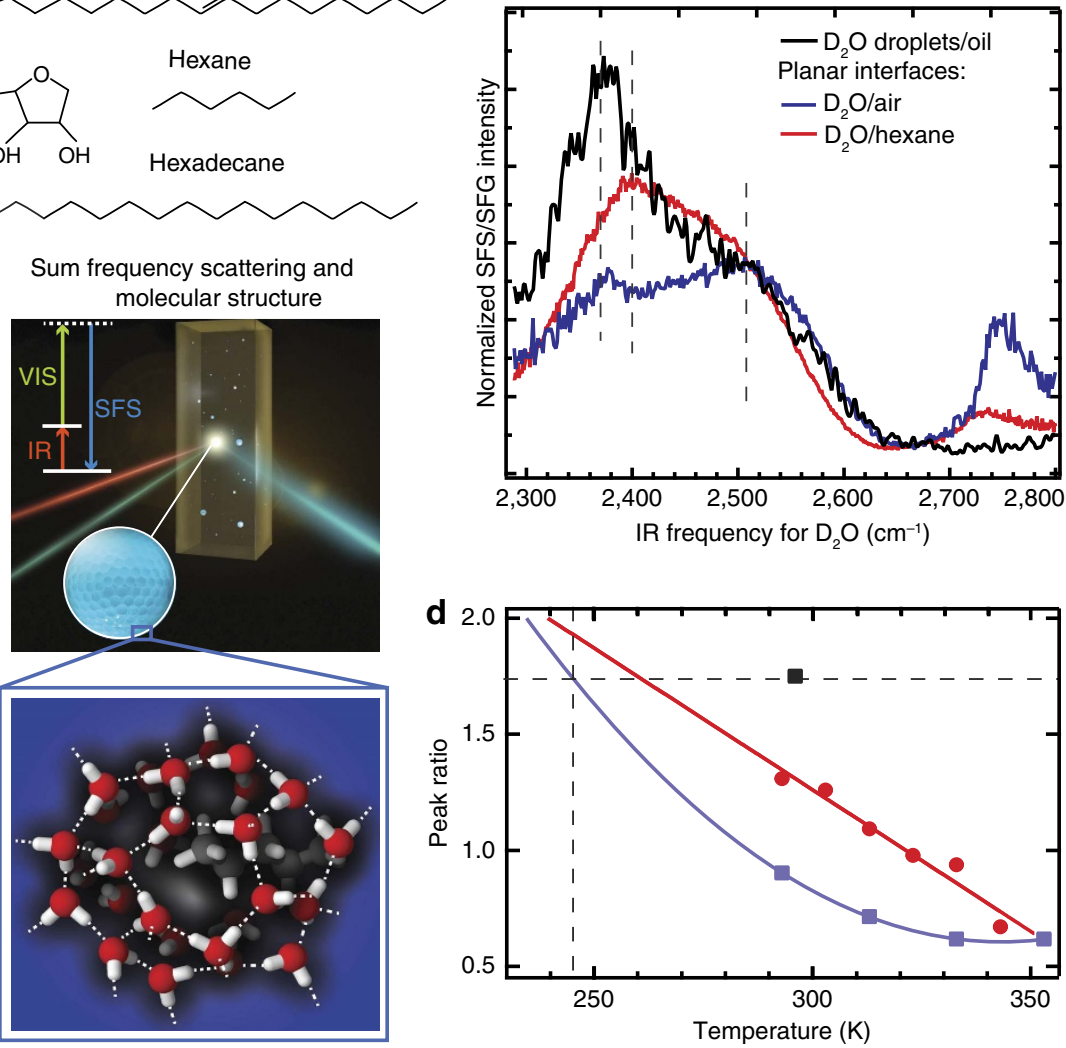

Figure 1 | Surface structure of water droplets. (a) Chemical structure of hexadecane, hexane and Span80. (b) Illustration of a vibrational SFS experiment. A femtosecond infrared (IR) and a picosecond visible (VIS) laser beam overlap in space and time in the sample and generate scattered sum frequency light from the water droplets. The inset illustrates the surface hydrogen-bond structure of a water droplet embedded in a hydrophobic liquid. In this figure, we sketch a possible molecular arrangement of the water molecules at the surface of a droplet surrounding an alkane tail that protrudes into the water phase (looking from the inside of the droplet). Please note that the surfactant is not present in the illustration. (c) SFS spectra of $D_{2} \mathrm{O}$ droplets in $d_{34}$-hexadecane with $5 \mathrm{mM}$ Span80 (black) and reflection SFG spectra of the planar $\mathrm{D}_{2} \mathrm{O} /$ air interface (blue) and planar $\mathrm{D}_{2} \mathrm{O} /$ hexane interface (red). The SFS/SFG spectra are collected with horizontally ( $P$ ) polarized infrared and vertically polarized (S) VIS and SF beams. The normalization procedure takes into account the infrared pulse shape in the sample as well as discontinuities of the electromagnetic fields at the interface (see Methods section). The top axis shows the corresponding frequencies axis for $\mathrm{H}_{2} \mathrm{O}\left(\times 1.35^{15}\right)$. (d) The ratio between the low- and high-frequency bands of the SFG spectrum of the planar water/air and water/hexane interface ${ }^{26}$ (blue and red markers) and an extrapolation with a quadratic polynomial fit to a lower temperature range (blue and red lines). The ratio for the room temperature water nanodroplet spectrum is shown as a black marker.

hydrophobic phase the water spectrum does not show a significant difference. In addition, reducing the liquid temperature below $273 \mathrm{~K}$ does not result in any spectral changes. Figure $2 \mathrm{~b}$ shows the SF spectrum of $\mathrm{D}_{2} \mathrm{O}$ nanocrystals $(233 \mathrm{~K})$ and the ice/ air interface for different temperatures $(170,200$ and $230 \mathrm{~K})$ in the frequency range around the strongly $\mathrm{H}$-bonded mode at $\sim 2,350(3,170) \mathrm{cm}^{-1}$. The resonance in the SF spectrum of the ice nanocrystals has a similar frequency as is observed for the planar ice surface of the same temperature. This indicates that the difference in surface structure observed for the liquid interface is not present in the solid phase. The absence of the effect for the frozen droplets can be understood from the fact that when the droplets freeze, the mutual water-water interactions become relatively more important for the resulting water SFG spectrum (and the detected H-bond strength) than the interactions with the hydrophobic oil molecules. For crystalline planar ice, the observed SF ice/air spectrum originates in part from $\mathrm{OH}$ groups connecting the different layers in the ice crystal $^{38}$. These deeper layers of water molecules are much less influenced by the hydrophobic groups at the interface. Hence, for planar ice and frozen water droplets of the same temperature the H-bonds of the probed $\mathrm{OH}$ groups are of similar strength.

\section{Discussion}

Thermodynamic ${ }^{39,40}$ and Raman multivariate curve resolution (MCR) measurements on solvated alkanols ${ }^{17}$ show water molecules hydrating a hydrophobic solute are more tetrahedrally ordered and thus more constrained in configurational space than water molecules in bulk liquid water (as sketched in the inset of Fig. 1b). These solutes are very small and fulfil the criterion of Chandler ${ }^{4}$ that states that hydrophobic solutes/surfaces lead to an enhancement of the tetrahedral ordering of water if the radius of the solute/surface is $<1 \mathrm{~nm}$ (ref. 4). In this perspective, the present observations of an enhanced ordering of water molecules at the surface of water nanodroplets embedded in oil is perhaps surprising, as the droplet radius $(100 \mathrm{~nm})$ is much larger than $1 \mathrm{~nm}$. However, we can consider the oil surrounding the water droplet surface as an extended curved array of hydrophobic perturbations, that is, hydrophobic groups protruding into the surface of the water droplet. The water molecules near the hydrophobic protrusions will fold their H-bond network around these $(<1 \mathrm{~nm})$ protrusions. We speculate that a high density of hydrophobic protrusions in combination with a modest overall curvature of the water surface (corresponding to a diameter of a few hundred 
a

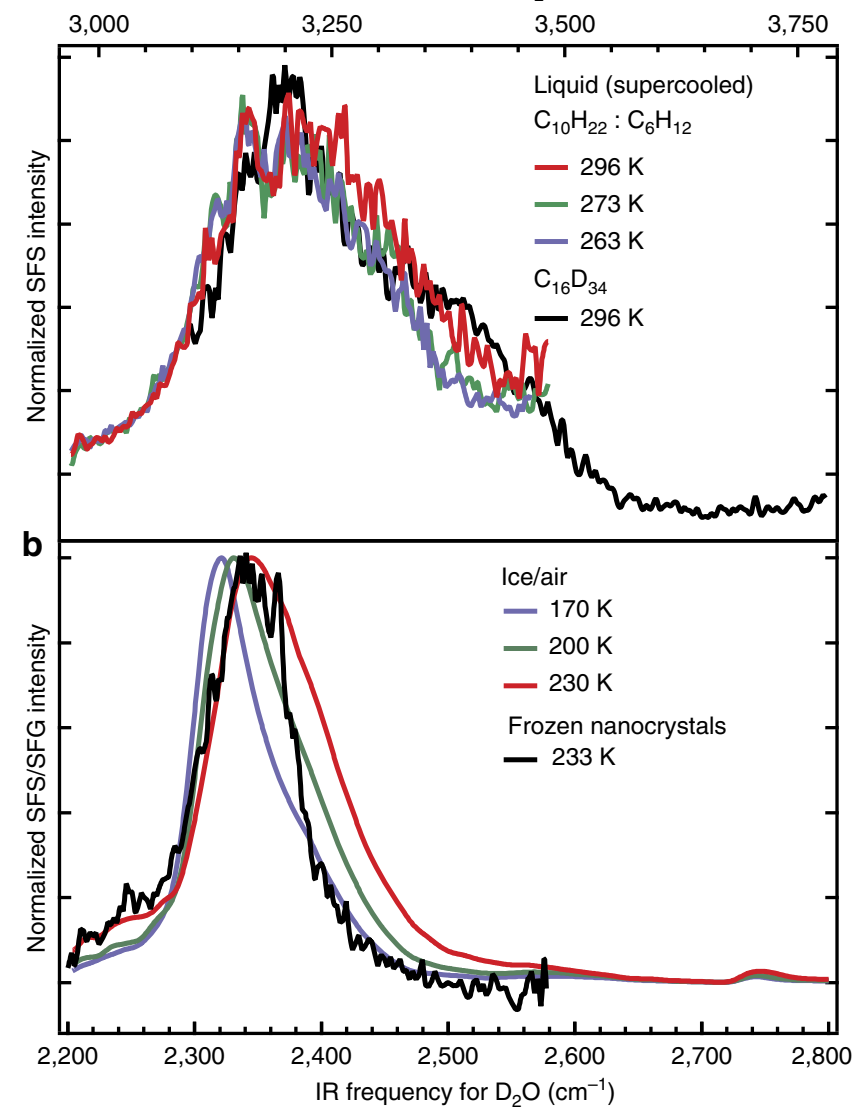

Figure 2 | Supercooled water droplets and ice nanocrystals. (a) SFS spectra of $D_{2} \mathrm{O}$ droplets at room temperature (296 K, in a mixture of decane and cyclohexane (red) and in $\mathrm{d}_{34}$-hexadecane (black)) and under supercooled conditions (green, $273 \mathrm{~K}$ and blue, $263 \mathrm{~K}$, both in a mixture of decane and cyclohexane). (b) SFS spectra of $\mathrm{D}_{2} \mathrm{O}$ droplets at $233 \mathrm{~K}$, below the homogeneous ice nucleation temperature (black) and SFG spectra of the basal $\mathrm{H}_{2} \mathrm{O}$ /air interface at $170 \mathrm{~K}$ (blue), $200 \mathrm{~K}$ (green) and $230 \mathrm{~K}$ (red). All droplets samples contain $5 \mathrm{mM}$ Span80. Spectral data from $\mathrm{H}_{2} \mathrm{O}$ and $\mathrm{D}_{2} \mathrm{O}$ are interchangeable. Frequency conversion between $\mathrm{H}_{2} \mathrm{O}$ and $\mathrm{D}_{2} \mathrm{O}$ was obtained by multiplying the $\mathrm{D}_{2} \mathrm{O}$ frequencies by $1.35^{15}$. The SFG spectra are collected with horizontally $(P)$ polarized infrared and vertically (S) polarized VIS and SF beams.

nanometres), leads to a strong structuring of the water hydrogenbond network, resulting in an extended highly structured corrugated droplet surface. For smaller droplets (for example, within micelles/solutes), the surface is too strongly curved to display such a favourable interplay between short-range and longrange interactions. For larger droplets, and flat surfaces, the curvature is too weak to get such a favourable interplay, leading to local disruptions of the $\mathrm{H}$-bond network and an enhanced inhomogeneity. This picture of the enhanced ordering of curved water surfaces will require support from high-quality molecular dynamics simulations.

In summary, vibrational SFS spectra from $100 \mathrm{~nm}$ radius water droplets embedded in a hydrophobic liquid at different temperatures show that the $\mathrm{H}$-bond network of the interfacial water possesses a much enhanced (in-plane) tetrahedral structure compared to a planar air/water interface. This greater order manifests itself as a red-shifted spectrum that has a $100 \%$ increased peak ratio of the 2,370 and $2,500 \mathrm{~cm}^{-1}$ modes. This increase is much larger than observed in previous studies of planar liquid hydrophobic/water interfaces. The observed structure of the water droplet interface corresponds to that of an air/water interface that is $\sim 50 \mathrm{~K}$ colder. This increase in order is explained by the formation of an extended network of hydrophobic protrusions into the water droplet surface, which does not exist on planar interfaces and in solution. On supercooling the droplets, the surface spectrum does not change shape. Cooling the sample below the homogeneous ice nucleation temperature results in a spectrum with a single symmetric peak at a comparable frequency as that of a planar basal ice/air interface of the same temperature.

The presented experiments demonstrate the possibility of quantifying the interfacial structure of water droplets and illustrate the effect of nanoscopic hydrophobic surfaces on the $\mathrm{H}$-bond structure of water. With a reduction of the effective 'surface temperature' we expect that the reactivity of water droplets embedded in a hydrophobic environment ${ }^{41}$, such as in cloud droplets, atmospheric ice particles ${ }^{1}$, rocks ${ }^{3}$ or medicine carriers ${ }^{9}$ is lower than what one might think based on the actual temperature. Future studies on such nanoscopic systems may reveal important information on the role and reactivity of interfacial water in aerosol formation, protein folding, pore functioning $4,6,7,42$ and the charging and stabilization of hydrophobic interfaces.

\section{Methods}

Chemicals. Before use all glassware was cleaned with a 1:3 $\mathrm{H}_{2} \mathrm{O}_{2}: \mathrm{H}_{2} \mathrm{SO}_{4}$ solution, after which it was thoroughly rinsed with ultra-pure water $\left(\mathrm{H}_{2} \mathrm{O}\right.$, Milli-Q UF plus, Millipore, Inc., electrical resistance of $18.2 \mathrm{M} \Omega \mathrm{cm}$ ). Hexadecane (Fluka, 99.8\%), decane (Fluka, 99.8\%), cyclohexane (Sigma, > 99.7\%), Span80 (Sigma, GC quality), Tween80 (Sigma, GC quality), sodium dodecyl sulfate, SDS (99\% BioMol), $\mathrm{d}_{34}$-hexadecane $\left(\mathrm{C}_{16} \mathrm{D}_{34}, 98 \% \mathrm{~d}\right.$, Cambridge Isotope Laboratories), fluorinated oil (Novec HFE7500, 3-Ethoxy-dodecafluor-2-trifluormethyl-hexan), sulfuric acid (95-97\%, ISO, Merck) and $\mathrm{H}_{2} \mathrm{O}_{2}(30 \%$, Reactolab SA) were used as received. The purity of alkanes was verified with a Zisman test ${ }^{43,44}$. All samples for SFS measurements were prepared using $\mathrm{D}_{2} \mathrm{O}(99.8 \%$ Armar, $>2 \mathrm{M} \Omega \mathrm{cm})$. The $\mathrm{D}_{2} \mathrm{O}$ for the water/air SFG experiment was obtained from Cambridge Isotope Laboratories (99.9\%).

Water nanodroplets. Water nanodroplets were prepared using a sonication procedure with 1 v.v.\% $\mathrm{D}_{2} \mathrm{O}$ in oil concentration and $5 \mathrm{mM}$ Span80 (or Span80:Tween 80 mixture), according to the procedure described in ref. 45. For some samples (shown in Supplementary Fig. 2) $10 \mathrm{mM}$ SDS was added to the water phase before the emulsification. The droplet-size distribution was measured with dynamic light scattering (DLS, Zetasizer Nano ZS, Malvern). For all samples, the droplets have a mean diameter of $\sim 200 \mathrm{~nm}$ with a polydispersity index of $\sim 0.2$. Infrared spectra were recorded with a Bruker Vertex 70 instrument equipped with silver mirrors.

Monocrystalline ice. Monocrystalline ice was grown following the manual of Basu et al. ${ }^{46}$. Five pieces of PVC pipe with small notches at the bottom were placed in an aluminium pan, which was chilled from underneath with a cooling liquid. Millipore water $(18.2 \mathrm{M} \Omega \mathrm{cm})$ was degassed and added until the bottoms of the moulds were covered. The temperature in the pan was set to $-0.5^{\circ} \mathrm{C}$ and after equilibration ice nucleation was initiated by adding a small piece of ice to the centre of the pan. The small notches at the bottom of each PVC mould allow only one ice crystal to propagate through, resulting in a single ice crystal in each mould. For 3 subsequent days, $1 \mathrm{~cm}$ water was added to each mould and the temperature was lowered by $0.3^{\circ} \mathrm{C}$. At day 4 , the ice was pushed outside of the pipe pieces and stored in a freezer, where all further handling was done. The crystal orientation was determined using a Rigsby-type universal stage $e^{47}$ and cut to the basal plane with a band saw. The ice surface was polished by a microtome and a $4 \mathrm{~mm}$ thick slice was cut off for the experiment.

Vibrational sum frequency reflection mode spectra. The SFG and VIS beams were polarized in the vertical $(S)$ direction, and the infrared beam was polarized in the horizontal plane (P), leading to the SSP (SFG, VIS, IR) polarization combination using the experimental set-up described in ref. 26. The set-up was purged with $\mathrm{N}_{2}$ gas. The VIS beam with a pulse energy of $14 \mu \mathrm{J}$ was centred at $798.6 \mathrm{~nm}$ with a full-width at half-maximum (FWHM) bandwidth of $13 \mathrm{~cm}^{-1}$. The $\mathrm{D}_{2} \mathrm{O} /$ air measurements were performed with the $10 \mu \mathrm{J} 300 \mathrm{~cm}^{-1}$ FWHM infrared beam centred at a wavelength of $4 \mu \mathrm{m}$. The procedure for measuring hexane/water is given in ref. 26. To measure the water/Span 80 interface, $0.16 \mu \mathrm{M}$ Span 80 was dissolved in $0.1 \mathrm{ml}$ hexane and added to liquid $\mathrm{D}_{2} \mathrm{O}$. Subsequently, the hexane was 
evaporated off. The diameter of the teflon tray for this experiment was $3.5 \mathrm{~cm}$. The basal ice/air measurements were performed with a $3 \mu \mathrm{J} 550 \mathrm{~cm}^{-1}$ FWHM IR beam centred at $3 \mu \mathrm{m}$. The angles of incidence with respect to the surface normal were $35^{\circ}$ (VIS) and $40^{\circ}$ (infrared). The $\mathrm{D}_{2} \mathrm{O} /$ air and basal ice spectra were recorded with acquisition times of $30 \mathrm{~s}$ and $300 \mathrm{~s}$, respectively. The spectral intensities were baseline subtracted and normalized with the infrared and VIS pulse energies, and the (normalized) SFG spectrum recorded from z-cut quartz.

The ice measurements were performed using a temperature cell that was cooled with liquid $\mathrm{N}_{2}$. It allows transmittance of the laser beams through a $\mathrm{CaF}_{2}$ window (see Supplementary Fig. 4). The temperature was monitored by a thermocouple welded on the edge of the ice surface with a drop of water and the desired temperature was set by a heating foil resistance, covered by a copper plate.

Vibrational SFS spectra. Vibrational SFS spectra were recorded using the set-up for SFG experiments described in refs $48-50$. An $800 \mathrm{~nm}$ regeneratively amplified Ti:sapphire system (Spitfire Pro, Spectra physics) seeded with an $80 \mathrm{MHz} 800 \mathrm{~nm}$ oscillator (Integral 50, Femtolasers) was operated at a $1 \mathrm{kHz}$ repetition rate to pump a commercial OPG/OPA/DPG system (HE-TOPAS-C, Light Conversion), which was used to generate infrared pulses. The visible beam was split off directly from the amplifier, and spectrally shaped with a home-built pulse shaper. The angle between the $10 \mu \mathrm{J}$ visible (VIS) beam $\left(800 \mathrm{~nm}\right.$, FWHM $\left.15 \mathrm{~cm}^{-1}\right)$ and the $6 \mu \mathrm{J}$ infrared beam $\left(3-4.5 \mu \mathrm{m}\right.$, FWHM $160 \mathrm{~cm}^{-1}$ ) was $20^{\circ}$ (as measured in air). The focused laser beams were overlapped in a sample cuvette with a path length of $200 \mu \mathrm{m}$. At a scattering angle $\left(\theta\right.$, measured in air) of $55^{\circ}$, the scattered sum frequency (SF) light was collimated using a plano-convex lens $(f=15 \mathrm{~mm}$, Thorlabs LA1540-B) and passed through two short-wave pass filters (3rd Millenium, 3RD770SP). The SF light was spectrally dispersed with a monochromator (Acton, SpectraPro 2300i) and detected with an intensified CCD camera (Princeton Instruments, PI-Max3) using a gate width of $10 \mathrm{~ns}$. The acquisition time for a single spectrum was $300 \mathrm{~s}$. A Glan-Taylor prism (Thorlabs, GT15-B), a half-wave plate (EKSMA, 460-4215) and a polarizing beam splitter cube (CVI, PBS-800-050) and two $\mathrm{BaF}_{2}$ wire grid polarizers (Thorlabs, WP25H-B) were used to control the polarization of the SFG, VIS and infrared beams, respectively. All measurements were performed in the SSP polarization combination. For normalization purposes, a reflection beam path is accessible by removable optics so that scattering and reflection can be done without a realignment procedure.

Temperature-controlled SFS measurements. Temperature-controlled SFS measurements were performed using a custom-made sample cell (Quantum Northwest, Supplementary Fig. 5). The sample cuvette was placed in a metallic holder cooled with a single Peltier element. The sample chamber was closed and filled with $\mathrm{N}_{2}$ gas to avoid condensation of air. The windows of the temperature cell were made from $\mathrm{CaF}_{2}$ (for the incoming beams) and quartz (for the scattered SF beam). The temperature cell itself was thermally isolated and also placed inside a box purged with $\mathrm{N}_{2}$ gas. The hot side of the Peltier element was cooled with a flow of cold liquid ethanol (223 K, using a peristaltic pump, Ismatec ISM1200). The cold ethanol was obtained by cooling ethanol inside a copper coil that was placed in a bath containing a mixture of dry ice and ethanol. The infrared beam path outside the laser source was purged with $\mathrm{N}_{2}$ gas.

Generation of broadband infrared pulses. To probe the whole frequency range of the $\mathrm{D}_{2} \mathrm{O}$ stretch mode region for the SFS spectral recordings, we stepped the infrared frequency trough the spectral window between 2,000 and $3,400 \mathrm{~cm}^{-1}$ using $50 \mathrm{~nm}$ steps (leading to a total acquisition time of 5,400 s per spectrum). Supplementary Fig. 6a shows SF spectra for the different infrared centre frequencies recorded in reflection mode from a z-cut quartz crystal. The summed spectrum for one of the runs is shown in Supplementary Fig. 6b.

Spectral shape and infrared absorption. The scattered SF spectra recorded from the water droplets were baseline subtracted and summed to obtain the spectral intensity for the entire spectral region of the OD stretching mode. The resulting spectrum was then divided by the energies of the infrared and VIS beams and normalized to the summed SFG spectrum of a z-cut quartz crystal (shown for example in Supplementary Fig. 6b). Since SF scattering experiments are performed in transmission mode, we have to take into account that the oil phase is absorbing part of the infrared spectrum, leading to a transmission spectrum $\mu_{\mathrm{FTIR}}$. To correct for absorption effects, we divide the obtained SFS spectrum by a Fourier transform infrared transmittance spectrum measured from the same sample in the same cuvette. Supplementary Fig. 7 shows the results of this correction as the blue spectra. It can be seen that this procedure results in significant spectral changes in the red spectral side since here the SFG spectrum overlaps with the CD modes of the deuterated main phase $\left(\mathrm{d}_{34}\right.$-hexadecane). The CH modes in the SFS spectrum originate from the Span 80 molecules that are present on the oil side of the interface (Span80 is insoluble in water ${ }^{18}$ ).

Discontinuity in the interfacial electric fields. Frequency-dependent refraction modifies the incident electromagnetic field at the interface, which can lead to significant frequency-dependent distortions in the obtained water spectra ${ }^{51,52}$. The sum frequency reflection mode spectra are therefore divided by the Fresnel factors ${ }^{14}$, whereby we use the refractive indices of $\mathrm{D}_{2} \mathrm{O}$ and ice from refs 53-55. The effective refractive index of the interfacial layers is calculated using a simple slab model ${ }^{56}$. For linear light scattering experiments such effects are captured by linear Mie theory ${ }^{57}$ and thus for SFS experiments by nonlinear Mie theory ${ }^{58}$. Using nonlinear Mie theory, we calculated a frequency-dependent correction factor for the SFS spectra using the input parameters from Supplementary Table 2. The refractive index values for oil and $\mathrm{D}_{2} \mathrm{O}$ are taken from refs 59,60. Supplementary Fig. 8 shows the effects of the correction on the spectral data reported in Figs 1 and 2 , where it can be seen that the influence of refraction effects is minimal. The reason for the small difference is that the droplets are small compared to the wavelength. For simplicity, in the main text we refer to the corrected SFS intensity $I_{\mathrm{SFS}} / I_{\mathrm{IR}} \mu_{\mathrm{FTIR}}$ as normalized SFS intensity.

Data availability. The data that support the findings of this study are available from the corresponding author on reasonable request.

\section{References}

1. de Leeuw, G. et al. Production flux of sea spray aerosol. Rev. Geophys. 49, 1-39 (2011).

2. Putnis, A. Why mineral interfaces matter. Science 343, 1441-1442 (2014).

3. Zachara, J. et al. Internal domains of natural porous media revealed: critical locations for transport, storage, and chemical reaction. Environ. Sci. Technol. 50, 2811-2829 (2016).

4. Chandler, D. Interfaces and the driving force of hydrophobic assembly. Nature 437, 640-647 (2005).

5. Zhang, L., Chen, K., Chen, B., White, J. L. \& Resasco, D. E. Factors that determine zeolite stability in hot liquid water. J. Am. Chem. Soc. 137, 11810-11819 (2015).

6. Dyson, H. J., Wright, P. E. \& Scheraga, H. A. The role of hydrophobic interactions in initiation and propagation of protein folding. Proc. Natl Acad. Sci. 103, 13057-13061 (2006).

7. Ball, P. Water as an active constituent in cell biology. Chem. Rev. 108, 74-108 (2008).

8. Möhlmann, D. T. F. Are nanometric films of liquid undercooled interfacial water bio-relevant? Cryobiology 58, 256-261 (2009).

9. Amstad, E., Kim, S.-H. \& Weitz, D. A. Photo- and thermoresponsive polymersomes for triggered release. Angew. Chem. Int. Ed. 51, 12499-12503 (2012).

10. Grzybowski, B. A. \& Huck, W. T. S. The nanotechnology of life-inspired systems. Nat. Nanotechnol. 11, 585-592 (2016).

11. Johnson, C. M. \& Baldelli, S. Vibrational sum frequency spectroscopy studies of the influence of solutes and phospholipids at vapor/water interfaces relevant to biological and environmental systems. Chem. Rev. 114, 8416-8446 (2014).

12. McFearin, C. L., Beaman, D. K., Moore, F. G. \& Richmond, G. L. From Franklin to today: toward a molecular level understanding of bonding and adsorption at the oil-water interface. J. Phys. Chem. C 113, 1171-1188 (2009).

13. Gan, W., Wu, D., Zhang, Z., Feng, R.-R. \& Wang, H.-F. Polarization and experimental configuration analyses of sum frequency generation vibrational spectra, structure, and orientational motion of the air/water interface. J. Chem Phys. 124, 114705 (2006).

14. Wang, H.-F., Gan, W., Lu, R., Rao, Y. \& Wu, B.-H. Quantitative spectral and orientational analysis in surface sum frequency generation vibrational spectroscopy (SFG-VS). Int. Rev. Phys. Chem. 24, 191-256 (2005).

15. Bonn, M., Nagata, Y. \& Backus, E. H. G. Molecular structure and dynamics of water at the water-air interface studied with surface-specific vibrational spectroscopy. Angew. Chem. Int. Ed. 54, 5560-5576 (2015).

16. Shen, Y. R. \& Ostroverkhov, V. Sum-frequency vibrational spectroscopy on water interfaces: polar orientation of water molecules at interfaces. Chem. Rev. 106, 1140-1154 (2006).

17. Davis, J. G., Gierszal, K. P., Wang, P. \& Ben-Amotz, D. Water structural transformation at molecular hydrophobic interfaces. Nature 491, 582-585 (2012).

18. Peltonen, L. J. \& Yliruusi, J. Surface pressure, hysteresis, interfacial tension, and $\mathrm{CMC}$ of four sorbitan monoesters at water-air, water-hexane, and hexane-air interfaces. J. Colloid Interface Sci. 227, 1-6 (2000).

19. Abraham, M. H., Whiting, G. S., Fuchs, R. \& Chambers, E. J. Thermodynamics of solute transfer from water to hexadecane. J. Chem. Soc. Perkin Trans. 2, 291-300 (1990).

20. Roke, S. et al. Vibrational sum frequency scattering from a submicron suspension. Phys. Rev. Lett. 91, 258302 (2003).

21. Roke, S. \& Gonella, G. Nonlinear light scattering and spectroscopy of particles and droplets in liquids. Annu. Rev. Phys. Chem. 63, 353-378 (2012).

22. Hunt, J. H., Guyot-Sionnest, P. \& Shen, Y. R. Observation of C-H stretch vibrations of monolayers of molecules optical sum-frequency generation. Chem. Phys. Lett. 133, 189-192 (1987). 
23. Harris, A. L., Chidsey, C. E. D., Levinos, N. J. \& Loiacono, D. N. Monolayer vibrational spectroscopy by infrared-visible sum generation at metal and semiconductor surfaces. Chem. Phys. Lett. 141, 350-356 (1987).

24. Bohren, C. F. \& Huffman, D. R. Absorption and Scattering of Light by Small Particles (Wiley, 2007).

25. Du, Q., Superfine, R., Freysz, E. \& Shen, Y. R. Vibrational spectroscopy of water at the vapor/water interface. Phys. Rev. Lett. 70, 2313-2316 (1993).

26. Strazdaite, S., Versluis, J., Backus, E. H. G. \& Bakker, H. J. Enhanced ordering of water at hydrophobic surfaces. J. Chem. Phys. 140, 054711 (2014).

27. Auer, B. M. \& Skinner, J. L. Vibrational sum-frequency spectroscopy of the water liquid/vapor interface. J. Phys. Chem. B 113, 4125-4130 (2009).

28. Ni, Y. \& Skinner, J. L. Communication: vibrational sum-frequency spectrum of the air-water interface, revisited. J. Chem. Phys. 145, 031103 (2016).

29. Buch, V. et al. Sum frequency generation surface spectra of ice, water, and acid solution investigated by an exciton model. J. Chem. Phys. 127, 204710 (2007).

30. Sovago, M. et al. Vibrational response of hydrogen-bonded interfacial water is dominated by intramolecular coupling. Phys. Rev. Lett. 100, 173901 (2008).

31. Scatena, L. F., Brown, M. G. \& Richmond, G. L. Water at hydrophobic surfaces: weak hydrogen bonding and strong orientation effects. Science 292, 908-912 (2001).

32. Stiopkin, I. V. et al. Hydrogen bonding at the water surface revealed by isotopic dilution spectroscopy. Nature 474, 192-195 (2011).

33. Shultz, M. J., Schnitzer, C., Simonelli, D. \& Baldelli, S. Sum frequency generation spectroscopy of the aqueous interface: ionic and soluble molecular solutions. Int. Rev. Phys. Chem. 19, 123-153 (2000).

34. Nihonyanagi, S. et al. Accurate determination of complex $\chi(2)$ spectrum of the air/water interface. J. Chem. Phys. 143, 124707 (2015).

35. Zhang, Z., Piatkowski, L., Bakker, H. J. \& Bonn, M. Ultrafast vibrational energy transfer at the water/air interface revealed by two-dimensional surface vibrational spectroscopy. Nat. Chem. 3, 888-893 (2011).

36. Jena, K. C., Scheu, R. \& Roke, S. Surface impurities are not responsible for the charge on the oil/water interface: a comment. Angew. Chem. Int. Ed. 51, 12938-12940 (2012).

37. Stöckel, P., Weidinger, I. M., Baumgärtel, H. \& Leisner, T. Rates of homogeneous ice nucleation in levitated $\mathrm{H} 2 \mathrm{O}$ and D2O droplets. J. Phys. Chem. A 109, 2540-2546 (2005).

38. Li Barnett, I., Groenzin, H. \& Shultz, M. J. Hydrogen bonding in the hexagonal ice surface. J. Phys. Chem. A 115, 6039-6045 (2011).

39. Frank, H. S. \& Evans, M. W. Free volume and entropy in condensed systems iii. Entropy in binary liquid mixtures; partial molal entropy in dilute solutions; structure and thermodynamics in aqueous electrolytes. J. Chem. Phys. 13, 507-532 (1945)

40. Glew, D. N. Aqueous solubility and the gas-hydrates. The methane-water system. J. Phys. Chem. 66, 605-609 (1962).

41. Jung, Y. \& Marcus, R. A. On the theory of organic catalysis 'on water'. J. Am. Chem. Soc. 129, 5492-5502 (2007).

42. Aryal, P., Abd-Wahab, F., Bucci, G., Sansom, M. S. P. \& Tucker, S. J. A hydrophobic barrier deep within the inner pore of the TWIK-1 K2P potassium channel. Nat. Commun. 5, 4377 (2014).

43. Day, J. P. R. \& Bain, C. D. Ellipsometric study of depletion at oil-water interfaces. Phys. Rev. E 76, 041601 (2007).

44. Bigelow, W. C., Pickett, D. L. \& Zisman, W. A. Oleophobic monolayers. J. Colloid Sci. 1, 513-538 (1946).

45. Chen, Y., Jena, K. C., Lütgebaucks, C., Okur, H. I. \& Roke, S. Three dimensional nano 'Langmuir trough' for lipid studies. Nano Lett. 15, 5558-5563 (2015).

46. Basu, K. et al. Determining the ice-binding planes of antifreeze proteins by fluorescence-based ice plane affinity. J. Vis. Exp. 83, e51185 (2014).

47. Groenzin, H. Sum-Frequency Studies Of Single Crystalline Ice Ih. PhD thesis, Tufts University (2007).

48. de Aguiar, H. B., Samson, J.-S. \& Roke, S. Probing nanoscopic droplet interfaces in aqueous solution with vibrational sum-frequency scattering: a study of the effects of path length, droplet density and pulse energy. Chem. Phys. Lett. 512, 76-80 (2011).

49. de Aguiar, H. B., Scheu, R., Jena, K. C., de Beer, A. G. F. \& Roke, S. Comparison of scattering and reflection SFG: a question of phase-matching. Phys. Chem. Chem. Phys. 14, 6826-6832 (2012).
50. Scheu, R., Chen, Y., Subinya, M. \& Roke, S. Stern layer formation induced by hydrophobic interactions: a molecular level study. J. Am. Chem. Soc. 135, 19330-19335 (2013).

51. Pool, R. E., Versluis, J., Backus, E. H. G. \& Bonn, M. Comparative study of direct and phase-specific vibrational sum-frequency generation spectroscopy: advantages and limitations. J. Phys. Chem. B 115, 15362-15369 (2011).

52. Feng, R.-R., Guo, Y., Lü, R., Velarde, L. \& Wang, H.-F. Consistency in the sum frequency generation intensity and phase vibrational spectra of the air/neat water interface. J. Phys. Chem. A 115, 6015-6027 (2011).

53. Warren, S. G. \& Brandt, R. E. Optical constants of ice from the ultraviolet to the microwave: a revised compilation. J. Geophys. Res. 113, D14220 (2008).

54. Max, J.-J. \& Chapados, C. Isotope effects in liquid water by infrared spectroscopy. III. H2O and D2O spectra from 6000 to $0 \mathrm{~cm}^{-1}$. J. Chem. Phys. 131, 184505 (2009).

55. Kedenburg, S., Vieweg, M., Gissibl, T. \& Giessen, H. Linear refractive index and absorption measurements of nonlinear optical liquids in the visible and near-infrared spectral region. Opt. Mater. Express 2, 1588-1611 (2012).

56. Zhuang, X., Miranda, P. B., Kim, D. \& Shen, Y. R. Mapping molecular orientation and conformation at interfaces by surface nonlinear optics. Phys. Rev. B 59, 12632-12640 (1999).

57. Jackson, J. D. Classical Electrodynamics (Wiley, 1999).

58. de Beer, A. G. F. \& Roke, S. Nonlinear Mie theory for second-harmonic and sum-frequency scattering. Phys. Rev. B 79, 155420 (2009).

59. Bertie, J. E., Ahmed, M. K. \& Eysel, H. H. Infrared intensities of liquids. 5. Optical and dielectric constants, integrated intensities, and dipole moment derivatives of water and water-d2 at 22 degree C. J. Phys. Chem. 93, 2210-2218 (1989).

60. Tuntomo, A., Tien, C. L. \& Park, S. H. Optical constants of liquid hydrocarbon fuels. Combust. Sci. Technol. 84, 133-140 (1992).

\section{Acknowledgements}

This work is supported by the Julia Jacobi Foundation, and the European Research Council (Grant number 616305). We thank Prof. Esther Amstad for providing the sample of water droplets in fluorinated oil. Dion Ursem and Hinco Schoenmaker are acknowledged for technical support. Part of this work belongs to the research programme of the Netherlands Organisation for Scientific Research (NWO) and was performed at the research institute AMOLF.

\section{Author contributions}

N.S. and W.J.S. performed experiments, N.S., W.J.S., H.J.B. and S.R. interpreted the data and wrote the manuscript. S.R. conceived and supervised the work.

\section{Additional information}

Supplementary Information accompanies this paper at http://www.nature.com/ naturecommunications

Competing interests: The authors declare no competing financial interests.

Reprints and permission information is available online at http://npg.nature.com/ reprintsandpermissions/

How to cite this article: Smolentsev, N. et al. The interfacial structure of water droplets in a hydrophobic liquid. Nat. Commun. 8, 15548 doi: 10.1038/ncomms15548 (2017).

Publisher's note: Springer Nature remains neutral with regard to jurisdictional claims in published maps and institutional affiliations.

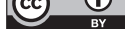

This work is licensed under a Creative Commons Attribution 4.0 International License. The images or other third party material in this article are included in the article's Creative Commons license, unless indicated otherwise in the credit line; if the material is not included under the Creative Commons license, users will need to obtain permission from the license holder to reproduce the material To view a copy of this license, visit http://creativecommons.org/licenses/by/4.0/

(C) The Author(s) 2017 